\title{
Expression and role of nestin in human cervical intraepithelial neoplasia and cervical cancer
}

\author{
ATSUKI SATO $^{1,2}$, TOSHIYUKI ISHIWATA ${ }^{1}$, YOKO MATSUDA ${ }^{1}$, TETSUSHI YAMAMOTO ${ }^{1}$, \\ HIROBUMI ASAKURA ${ }^{2}$, TOSHIYUKI TAKESHITA ${ }^{2}$ and ZENYA NAITO $^{1}$ \\ ${ }^{1}$ Departments of Pathology and Integrative Oncological Pathology, Nippon Medical School, Tokyo 113-8602; \\ ${ }^{2}$ Division of Reproductive Medicine, Perinatology and Gynecologic Oncology, \\ Graduate School of Medicine, Nippon Medical School, Tokyo 113-8603, Japan
}

Received January 25, 2012; Accepted March 2, 2012

DOI: $10.3892 /$ ijo.2012.1473

\begin{abstract}
Nestin expression reportedly correlates with aggressive growth, metastasis, poor prognosis and presence of cancer stem cells (CSCs) in various tumors. In this study, we determined the expression and role of nestin in cervical intraepithelial neoplasia (CIN) and cervical cancer. We performed immunohistochemical and in situ hybridization analyses of nestin in 26 cases for each stage of CIN and 55 cervical cancer tissue samples. To examine the role of nestin in cervical cancer cells, we stably transfected expression vectors containing nestin cDNA into ME-180 cells. We studied the effects of increased nestin expression on cell proliferation, cell motility, invasion as well as sphere and soft agar formation. Nestin was not localized in the squamous epithelium in normal cervical tissues, but it was weakly expressed in the basal squamous epithelium of CIN 1. In CIN 2, nestin was localized to the basal to lower $2 / 3$ of the squamous epithelium, whereas in CIN 3, it was localized to the majority of the squamous epithelium. Nestin was detected in all cases of invasive cervical cancer. Nestin mRNA was expressed in both ME-180 and CaSki cells. Growth rate, cell motility and invasion ability of stably nestin-transfected ME-180 cells were not different from empty vector-transfected ME-180 (mock cells). However, the nestin-transfected ME-180 cells formed more colonies and spheres compared to the mock cells. These findings suggest that nestin plays important roles in carcinogenesis and tumor formation of cervical cancer cells. Nestin may closely correlate with regulation of CSCs.
\end{abstract}

\section{Introduction}

Cervical cancer is the second most prevalent cancer in women worldwide and its occurrence has steadily increased among

Correspondence to: Professor Zenya Naito, Departments of Pathology and Integrative Oncological Pathology, Nippon Medical School, 1-1-5 Sendagi, Bunkyo-ku, Tokyo 113-8602, Japan

E-mail: naito@nms.ac.jp

Key words: nestin, cervical intraepithelial neoplasia, uterine cervical cancer, immunohistochemistry, ME-180, cancer stem cell young women $(1,2)$. Nearly half million new cases and 274,000 deaths occur each year due to this disease (3). More than $99 \%$ of cervical carcinomas are associated with human papillomavirus (HPV), yet infection of HPV alone is not sufficient to cause cervical cancer (4).

Nestin is a protein belonging to the class VI intermediate filaments (IFs). It was first described as a marker of neuroectodermal stem and progenitor cells because it is expressed in proliferating cells during embryonic development of the central nervous system (CNS) (5). Nestin is a large protein $(>1,600$ amino acids), structurally similar to other IFs, with a highly conserved $\alpha$-helical core domain of 300-330 amino acids flanked by amino- and carboxy-terminal domains $(5,6)$. Nestin contains a short $\mathrm{N}$-terminus and an unusually long $\mathrm{C}$-terminus. It is assembled into IF by forming heterodimers with vimentin and desmin, but nestin cannot form homopolymers (7-10).

Nestin is also expressed in immature or progenitor cells in non-neuronal tissues (11-14). Strong nestin expression has been detected in oligodendroglial lineage cells, ependymocytes, Sertoli cells, enteroglia, hair follicle cells, podocytes of renal glomeruli, stellate cells, pericytes, islets, optic nerve and odontoblasts (15-21). Various tumors show increased nestin expression, including central nervous system (CNS) tumors, melanomas, gastrointestinal stromal tumors (GIST), prostate cancer, breast cancer and pancreatic cancer (22-27). Nestin has been detected in human gliomas, with expression more frequent in high-grade gliomas than in low-grade gliomas such as pilocytic astrocytomas $(28,29)$. In malignant melanoma, stronger nestin expression is observed in advanced stage disease and in metastatic foci of melanoma cells $(24,30)$. Nestin protein is most abundant at the infiltrating front of the tumors, suggesting that it plays important roles in melanoma cell migration and invasion (31). In breast cancer, nestin expression is associated with shorter survival and is an independent prognostic factor (32). Approximately $30 \%$ of pancreatic ductal adenocarcinoma (PDAC) cases show nestin immunoreactivity; nestin expression correlates with perineural invasion and the presence of cancer cells at the tumor resection margins in PDAC (6). Our group recently reported that expression levels of nestin directly correlate with migration, invasion and metastasis of pancreatic cancer cells (33).

Nestin has also received attention as a cancer stem cell (CSC) marker in various tumor cells including CNS tumors, 
uterine and cervical cancer, prostate, bladder, head and neck, ovarian, testicular and pancreatic cancers as well as malignant rhabdoid tumors (34-41).

However, the expression and role of nestin in cervical intraepithelial neoplasia (CIN) and cervical cancer are poorly understood. Here we report the correlation of nestin expression pattern with CIN progression. We found that nestin was expressed in all cervical cancer specimens. Increased nestin expression in cervical cancer cell lines stimulated colony and sphere formation in vitro.

\section{Materials and methods}

Non-cancerous, CIN and cervical cancer tissues. Cervical cancer samples (55 patients; mean age 50.4 years; median age 50 years; range $29-72$ years) were obtained from patients undergoing surgery for invasive cervical cancer at the Department of Obstetrics and Gynecology of Nippon Medical School Hospital (Tokyo, Japan) from 2004-2010 (Table I). Twenty-six samples each for CIN 1-3 were obtained from cervical biopsies at the same hospital. For control samples, cervical biopsies from patients with chronic cervicitis (12 patients) were obtained. This study was carried out following the ethical guidelines of Nippon Medical School and the principles embodied in the Declaration of Helsinki, 2008.

Immunohistochemistry. Paraffin-embedded sections $(3 \mu \mathrm{m})$ were subjected to immunostaining using a Histofine Simple Stain MAX PO (M) kit (Nichirei Corporation, Tokyo, Japan) for nestin as previously reported (42). Tissue sections were incubated with the anti-nestin antibody (1:200 dilution; R\&D Systems, Westerville, OH) in phosphate-buffered saline (PBS) containing $1 \%$ bovine serum albumin (BSA) for $16 \mathrm{~h}$. Bound antibodies were detected with the Simple Stain MAX PO (M) reagent, using diaminobenzidine tetrahydrochloride as the substrate. Negative control tissue sections were prepared by omitting the primary antibody.

In situ hybridization analysis. A 235-bp BamHI-EcoRI cDNA fragment, corresponding to nucleotides 1045-1227 of the human nestin cDNA sequence, was subcloned into the pGEM-T vector and the result was confirmed by sequencing. Probes were labeled with digoxigenin-UTP using SP6 or T7 RNA polymerase and the DIG RNA-labeling kit (Roche Diagnostics GmbH, Mannheim, Germany). In situ hybridization was carried out as previously reported (43).

Human cervical cancer cell line. ME-180 cells were obtained from the Cell Resource Center for Biomedical Research,Institute of Development, Aging and Cancer (Tohoku University, Sendai, Japan). CaSki cells were obtained from RIKEN BioResource Center (Ibaraki, Japan). ME-180 cells were grown in RPMI1640 medium containing 10\% heat-inactivated fetal bovine serum (FBS) at $37^{\circ} \mathrm{C}$ under a humidified $5 \% \mathrm{CO}_{2}$ atmosphere.

Construction of nestin expression vector. The full-length nestin cDNA fragment was ligated to the 3 '-end of the human cytomegalovirus early promoter/enhancer in pAcGFP1-N1, a eukaryotic expression vector (Clontech Laboratories, Mountain View, CA). The correct orientation of the insert was verified by
Table I. Clinical features of cervical cancer patients.

\begin{tabular}{lr}
\hline Age (years) & $29-72$ \\
Median & 50 \\
Mean & 50.4 \\
FIGO stage & \\
Ia & \\
Ib & 30 \\
IIa & 3 \\
IIb & 14 \\
IIIa & 1 \\
IIIb & 3
\end{tabular}

Keratinization

Absent 34

Present 21

Nodal metastasis

Absent $\quad 37$

Present 18

HPV infection

Absent 3

Present 23

Not examined 29

${ }^{a}$ FIGO, International Federation of Gynecology and Obstetrics.

DNA sequencing. Nestin expressing and empty vectors were transfected using FuGENE HD transfection reagent (Roche Diagnostics $\mathrm{GmbH}$ ). For stably-transfected cells, the cells were passaged and cultured with $1,000 \mu \mathrm{g} / \mathrm{ml}$ of Geneticin.

Quantitative real-time PCR of nestin in cervical cancer cell lines. Cervical cancer cells were grown in RPMI-1640 medium with $10 \%$ FBS for $48 \mathrm{~h}$. Total-RNA extraction from cervical cancer cells was performed using a NucleoSpinII RNA kit (Takara Bio, Inc., Shiga, Japan). Then, cDNA synthesis was performed using High Capacity cDNA Reverse Transcription kit following the manufacturer's protocol (Applied Biosystems, Carlsbad, CA). Real-time PCR for nestin and 18S rRNA was performed using the StepOnePlus realtime PCR system (Applied Biosystems) with specific primers for nestin (Hs00707120_s1) and 18S rRNA (Hs03928990_g1) and a TaqMan probe (7).

Immunofluorescence staining and confocal laser microscopy. The same anti-nestin antibody used for the immunohistochemistry was also used for immunofluorescence staining of ME-180 cells. The cells were incubated with the anti-nestin antibody (1:50) in PBS containing 1\% BSA for $18 \mathrm{~h}$ at $4^{\circ} \mathrm{C}$. The cells were washed with PBS and then incubated with Alexa 568-conjugated goat anti-mouse IgG (Invitrogen Life Technologies, Carlsbad, CA) for $1 \mathrm{~h}$. Then, cells were mounted with Vectashield mounting medium containing DAPI (Vector Laboratories, Inc., Burlingame, CA). Fluorescent images were acquired using a confocal laser scanning microscope Digital Eclipse TE 2000-E (Nikon Instech Co., Ltd., Tokyo, Japan) and were analyzed using the confocal microscope Digital 
Eclipse C1 control software EZ-C1 (version 2.30) (Nikon Instech Co., Ltd.).

Morphological analysis of nestin-transfected ME-180 cells. Stably nestin-transfected ME-180 cells, empty-vectortransfected (mock cells) and nontreated cells (wild) were incubated in RPMI-1640 medium with $10 \%$ FBS for $48 \mathrm{~h}$. Cell morphology was observed using a phase-contrast microscope (Nikon Eclipse TE2000-U).

Anchorage-dependent growth assays. To examine the growth rates of nestin-transfected clones, a nonradioactive cell proliferation assay was performed. The cells were cultured in RPMI-1640 medium with $10 \%$ FBS at a density of $5 \times 10^{3}$ in 96-well plates followed by incubation at $37^{\circ} \mathrm{C}$ in a humidified $5 \% \mathrm{CO}_{2}$ atmosphere for 24, 48, 72, 96, 120 and $144 \mathrm{~h}$. Then, the cells were incubated with WST-8 cell counting reagent (Wako Pure Chemical Industries, Ltd., Osaka, Japan) for $4 \mathrm{~h}$ at $37^{\circ} \mathrm{C}$ and the optical density of the culture solution in the plate was measured using an ELISA plate reader (Bio-Rad, Hercules, CA) at $450 \mathrm{~nm}$. Alternatively, cells were plated at a density of $5 \times 10^{4}$ cells/well and the cell number of each well was counted after 24, 48, 72 and 96 h using C-reader (Digital Bio Technology Co., Ltd., Kyungki-do, Korea).

Growth of nestin-transfected ME-180 cells in soft agar. In vitro tumorigenicity was determined on the basis of cell growth in a soft agar colony assay. The flasks were covered with $2.5 \mathrm{ml}$ RPMI-1640/0.5\% agar/10\% FBS. The upper layer consisted of $2 \mathrm{ml} \mathrm{RPMI-1640/0.03 \%} \mathrm{agar/10 \%} \mathrm{FBS.} 1 \times 10^{3}$ and $2 \times 10^{3}$ cells/well were seeded and incubated under a humidified $5 \% \mathrm{CO}_{2}$ atmosphere at $37^{\circ} \mathrm{C}$ for 60 days. Images were obtained using Coolpix 5000 (Nikon Instech Co., Ltd.).

Single-cell movement assay. ME-180 cells (8,000/well) were seeded onto a 4 -well glass bottom dish and grown for $48 \mathrm{~h}$. Cell movement was then monitored for $24 \mathrm{~h}$ using a Digital Eclipse TE 2000-E motorized inverted microscope taking pictures every $5 \mathrm{~min}$. The total distance of individual cells covered within $24 \mathrm{~h}$ was determined using the Metamorph software 7.6 (Universal Imaging Corporation Ltd., Buckinghamshire, UK).

Cell invasion assays. To assess the effect of nestin on cervical cancer cell invasion, an in vitro invasion assay using a modified Boyden chamber technique was carried out. Matrigel-coated inserts $(8 \mu \mathrm{m}$ pore size and $6 \mathrm{~mm}$ in diameter; Becton-Dickinson and Co., Franklin Lakes, NJ) were used to assay cell invasion following the manufacturer's instructions. Briefly, cell were suspended in $500 \mu \mathrm{l}$ serum-free medium and placed onto the upper component of the inserts at a density of $1 \times 10^{5}$ cells. The lower compartment was filled with $750 \mu 1$ medium containing $10 \% \mathrm{FBS}$ and the cells were incubated at $37^{\circ} \mathrm{C}$ in a humidified $5 \% \mathrm{CO}_{2}$ atmosphere. After $48 \mathrm{~h}$, the cells on the upper surface of the filter were fixed and stained with a Diff-Quick staining kit and counted under a light microscope. The cell number on each membrane was counted in 5 highpower fields (magnification, x200).

Sphere formation assay. To determine whether cervical cancer cells had CSC-like characteristics, we performed sphere
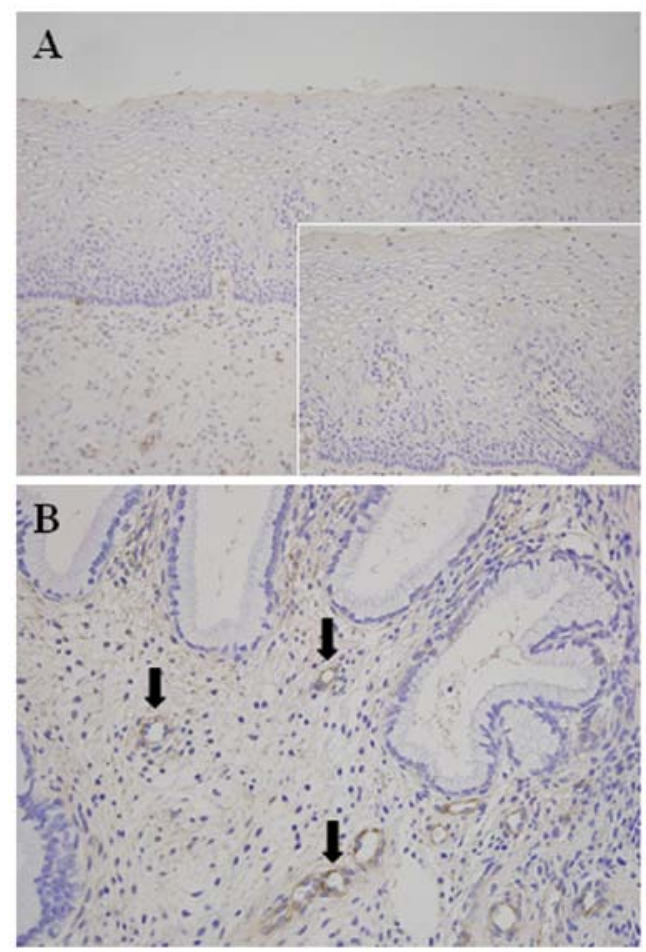

Figure 1. Immunohistochemical analysis of nestin in non-cancerous cervical tissues. (A and inset) Nestin protein was not localized in squamous epithelial cells, (B) but was localized in vascular endothelial cells. Original magnification, (A) x200, inset x400, (B) x400.

formation assays. ME-180 cells (1,000/well) were plated in ultra-low attachment surface 24 -well plate with serum-free medium supplemented with bFGF (10 ng/ml; ReproCell, Inc., Kanagawa, Japan) and EGF (20 ng/ml; R\&D Systems). After 7 days, the number of spheres in each well was counted by phase-contrast microscope (Eclipse TE2000-U).

Statistical analysis. All quantitative data are presented as mean \pm standard error (SE) and assessed using Student's t-test and Bonferroni/Dunn test. Computations were performed using the Stat View J version 5.0 software package (SAS Institute, Inc., Cary, NC).

\section{Results}

Immunohistochemical analysis of nestin in non-cancerous cervical tissue. Immunohistochemical analyses were performed to examine the localization of nestin protein in cervical tissues. In non-cancerous cervical tissue, nestin protein was not identified in squamous epithelial cells (Fig. 1A and inset). Nestin was not detected in cervical glands, whereas it was strongly expressed in stromal vascular endothelial cells (Fig. 1B).

Immunohistochemical and ISH analyses of nestin in CIN tissues. In CIN 1, nestin protein and mRNA were weakly expressed or absent in the basal to lower $1 / 3$ of squamous epithelial layers (Fig. 2A and D). In CIN 2, nestin protein and mRNA were localized between the basal layer and the lower 2/3 of squamous epithelial layers (Fig. 2B and E). In CIN 3, they were strongly expressed in most squamous epithelial 

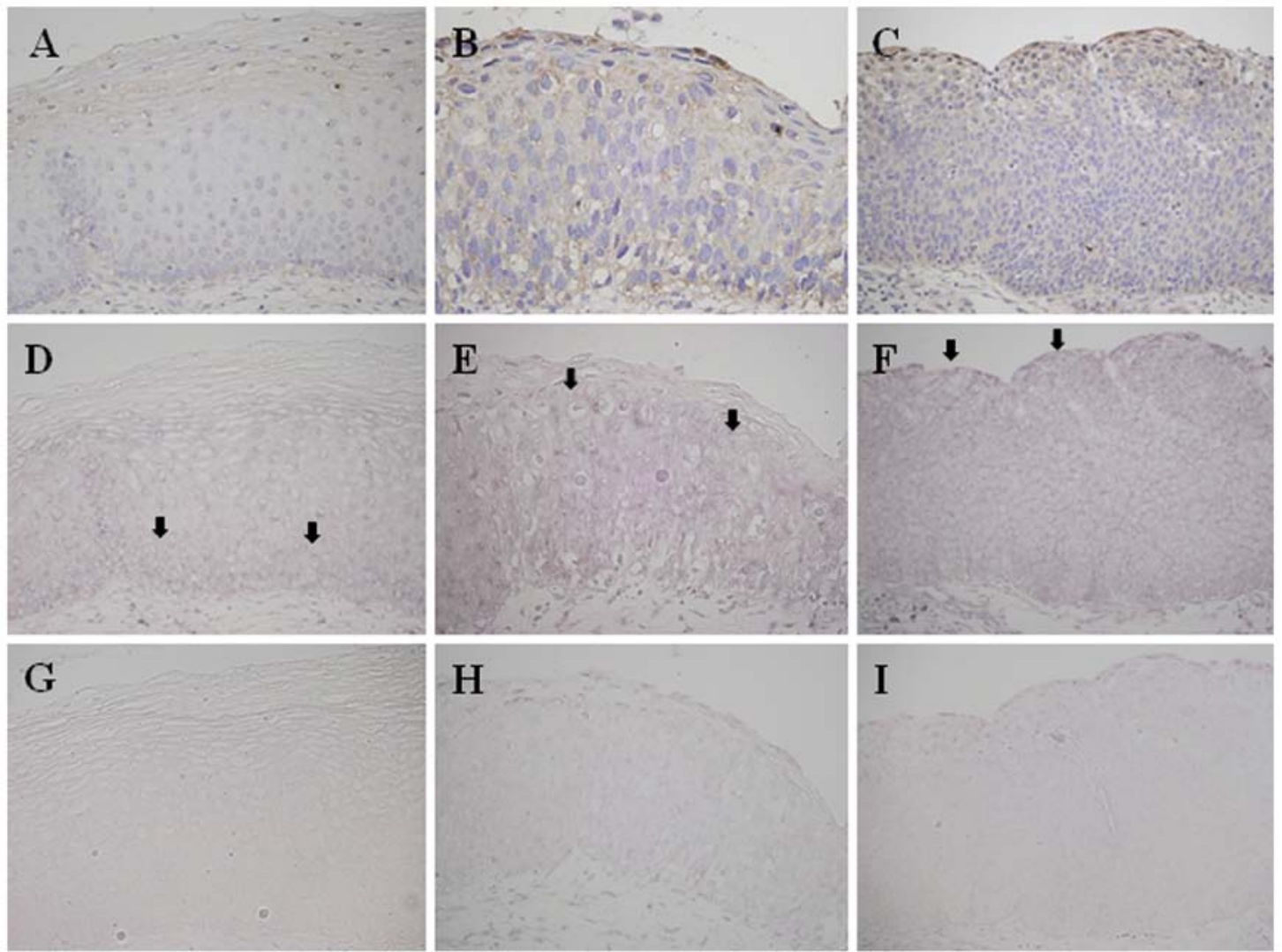

Figure 2. Immunohistochemical and ISH analyses of nestin in CIN. (A and D) Nestin protein and mRNA were localized at the basal to lower $1 / 3$ of squamous epithelial layers in CIN 1, (B and E) between the basal layer and the lower 2/3 in CIN 2 and (C and F) in most squamous epithelial layers in CIN 3. (A-C) Immunohistochemistry; (D-F) in situ hybridization, antisense; (G-I) sense. Original magnification, (A, B, D, E, G and H) x400 and (C, F and I) x300.
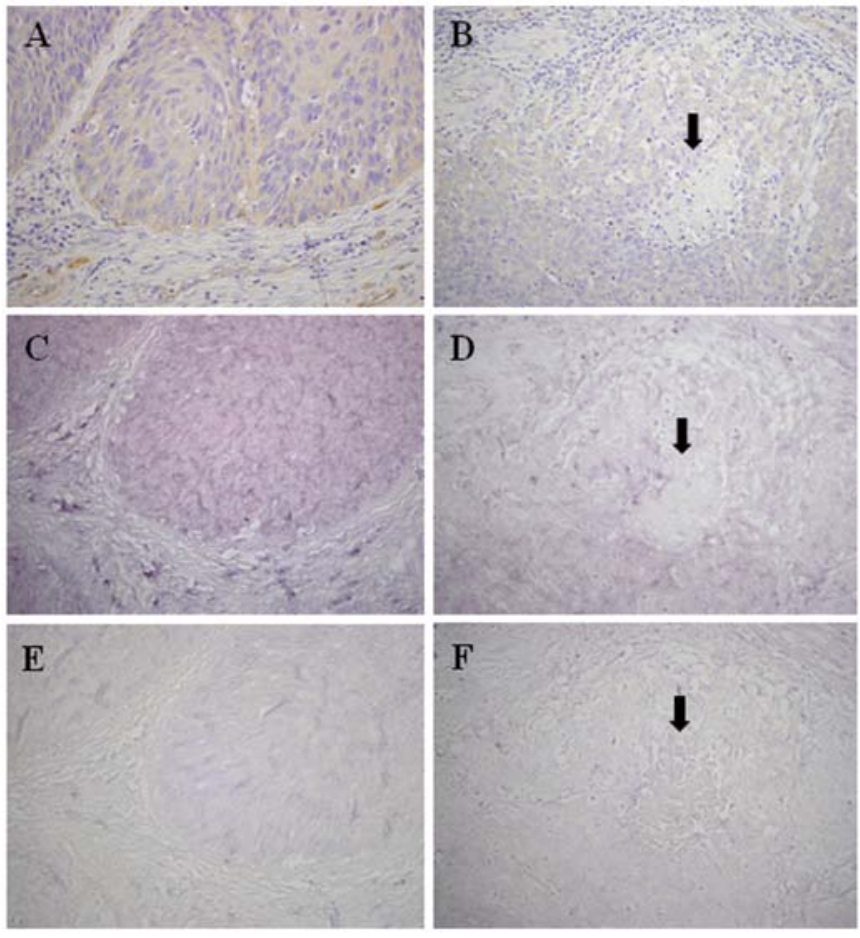

Figure 3. Immunohistochemical and ISH analyses of nestin in cervical cancer. (A, C and E) Nestin protein and mRNA were diffusely localized in cancer cell nests in the nonkeratinizing and $(\mathrm{B}, \mathrm{D}$ and $\mathrm{F})$ keratinizing tumor types. (A and B) Immunohistochemistry; (C and D) in situ hybridization, antisense; (E and F) sense. Arrows, keratinizing foci; original magnification, (A, C and E) $\times 200$ and (B, D and F) $\times 400$. layers (Fig. 2C and F). Sense probes did not show positive signals (Fig. 2G, $\mathrm{H}$ and I).

Immunohistochemical and ISH analyses of nestin in cervical cancer tissues. Nonkeratinizing cervical cancers showed solid and alveolar proliferative patterns, while keratinizing tumors showed foci of keratinization within nests of cancer cells (Fig. 3A and B). After mmunohistochemical analysis, nestin protein was detected in cancer cells of both the nonkeratinizing and keratinizing types in all examined cervical cancer samples. Nestin protein and mRNA were diffusely localized in cancer cell nests in the nonkeratinizing and keratinizing tumor cells (Fig. 3A-D). Nestin protein and mRNA were also expressed in endothelial cells of small-sized vessels near cancer cells. Nestin was not detected in the keratinizing areas within keratinizing types of cervical cancer (Fig. 3B and D). Sense probes did not show positive signals (Fig. 3E and F).

Expression levels of nestin in cervical cancer cell lines. To examine whether cervical cancer cells express nestin, Q-PCR was performed. Nestin mRNA was expressed in CaSki and ME-180 cervical cancer cell lines. Expression of nestin mRNA was 2.48-fold higher in CaSki cells than in ME-180 cells (Fig. 4A).

Stably nestin-transfected ME-180 cells. To examine the roles of nestin in cervical cancer cells, we prepared nestinoverexpressing ME-180 cells. Full-length nestin cDNA was 
A

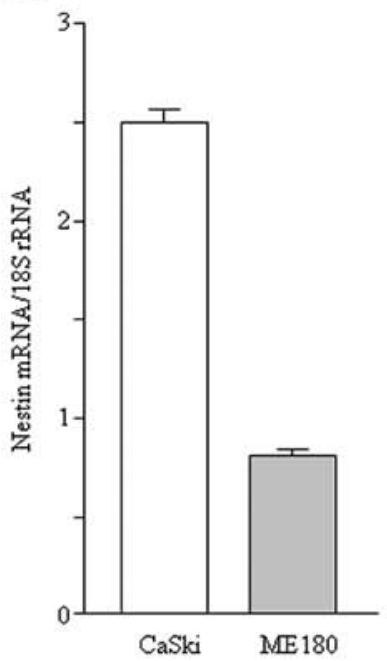

$\mathrm{B}$

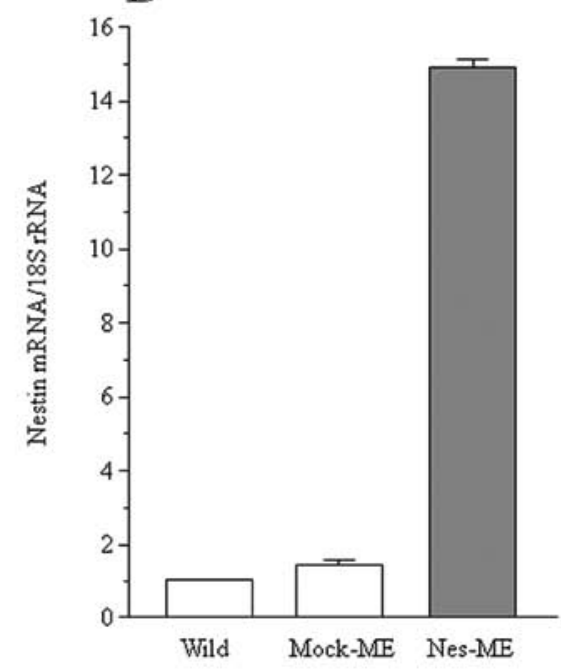

C
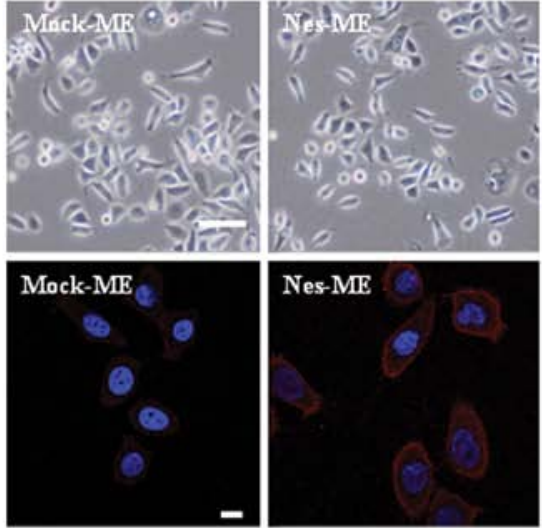

Nes-ME

Figure 4. Cervical cancer cell lines and transfection of nestin expression vector into ME-180 cells. (A) Expression level of nestin mRNA was lower in ME-180 than in CaSki cells. Nestin expression vector was stably transfected into ME-180 cells. (B and C) Nestin mRNA and protein levels were higher in nestintransfected ME-180 cells (Nes-ME) than in control cells. (C) Phase-contrast images: bar, 1,000 $\mu$ m; immunofluorescence images: bar, $20 \mu$ m.

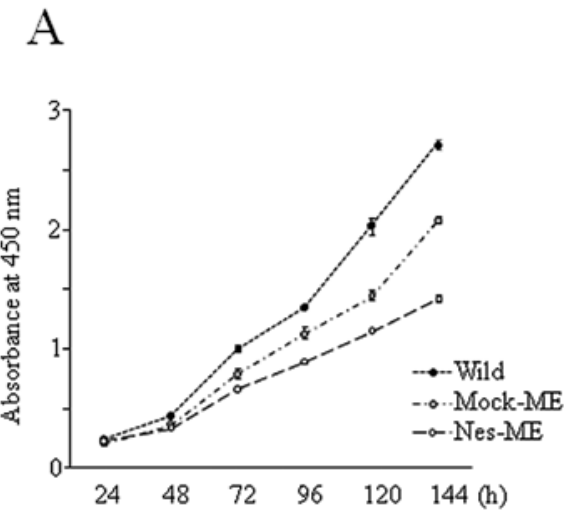

$\mathrm{B}$

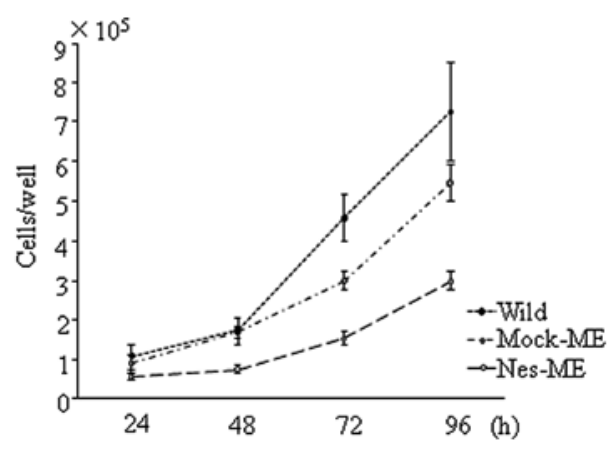

Figure 5. (A) Cell proliferation assay. The growth rates of Nes-ME cells using WST-8 reagent were lower than those of mock cells (Mock-ME), but this difference was not statistically significant. (B) Growth rates using cell count of Nes-ME cells were lower than those of Mock-ME cells, but the difference was not statistically significant.

subcloned into pAcGFP1-N1 vector and stably transfected in the cells. The expression levels of nestin mRNA in mock cells (Mock-ME) and nestin-transfected ME-180 cells (Nes-ME) were examined by Q-PCR. Nestin mRNA levels were high in
Nes-ME cells and low in Mock-ME and wild cells (Fig. 4B). Expression of nestin mRNA was 9.15-fold higher in Nes-ME than Mock-ME cells. Nes-ME cells did not show characteristic morphological alterations as compared with Mock-ME cells (Fig. 4C, upper panels). Immunofluorescent analysis showed that nestin protein is expressed in the cytoplasm of ME-180 cells, with stronger expression in Nes-ME than in Mock-ME cells (Fig. 4C, lower panels).

Anchorage-dependent growth and cell motility assays. Nes-ME and Mock-ME cells were cultured for 24, 48, 72, 96,120 and $144 \mathrm{~h}$. Growth rates of stable Nes-ME cells were lower than those of Mock-ME cells, but this difference was not statistically significant (Fig. 5A). Growth rates using cell count of Nes-ME cells were lower than those of Mock-ME cells, similarly with the non-radioactive cell proliferation assay using WST-8 reagent (Fig. 5B). Next, a single-cell movement assay using time-lapse microscopy was performed to determine if nestin is involved in cellular motility. There was no significant difference between Nes-ME and Mock-ME cells( Fig. 6A and B). We also performed a modified Boyden chamber assay to assess cell invasion. There was no difference in invasive ability between Mock-ME and Nes-ME cells (Fig. 6C).

Anchorage-independent growth assay. To examine the effect of increased expression of nestin in ME-180 cells on colony formation, a soft agar assay was performed. Nes-ME cells cultured with $1 \times 10^{3}$ (Fig. 7B) and $2 \times 10^{3}$ cells/well (Fig. 7A and $\mathrm{C}$ ) formed more colonies than Mock-ME and wild cells in 60 days $(\mathrm{P}<0.0001)$.

Sphere forming assay. To determine whether cervical cancer cells had characteristics of CSCs, a sphere formation assay was performed. Cells were plated in ultra-low attachment plates supplemented with EGF and bFGF, and then cultured for 7 days. Nes-ME cells formed larger size (Fig. 7D) and more numerous spheres than Mock-ME cells $(\mathrm{P}<0.0001)$ (Fig. 7E). 
A
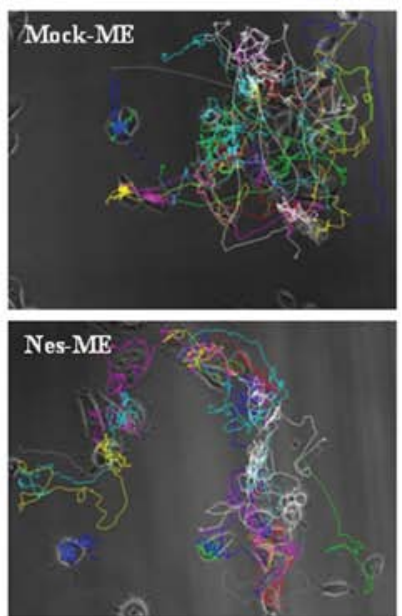

B

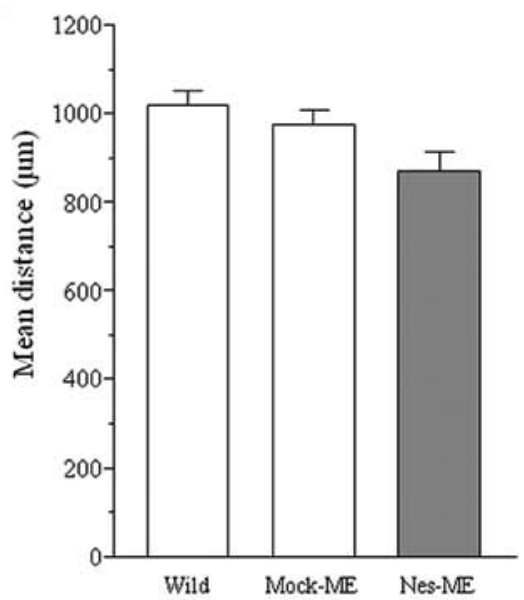

C

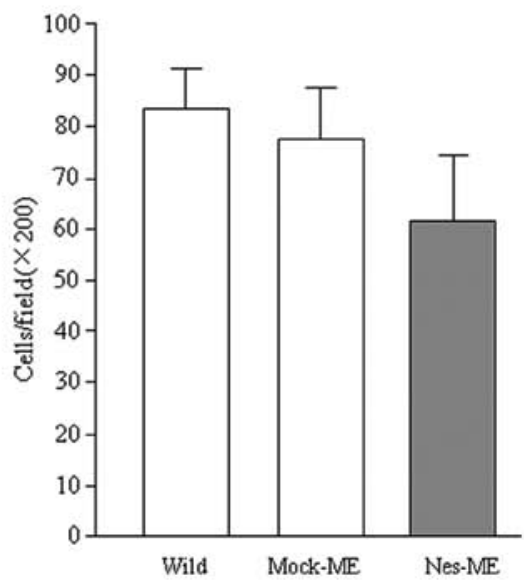

Figure 6. Cell motility assays. (A and B) The distances covered within $24 \mathrm{~h}$ by Mock-ME and Nes-ME cells showed no statistical difference. (C) In the invasion assay, Mock-ME and Nes-ME cells showed no difference in cell motility.

A

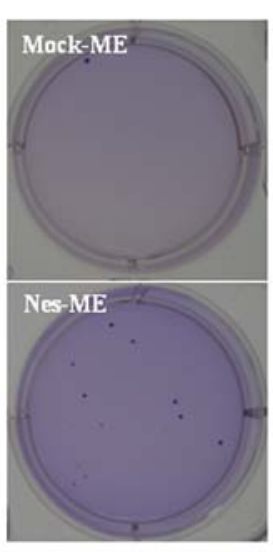

$\mathrm{B}$

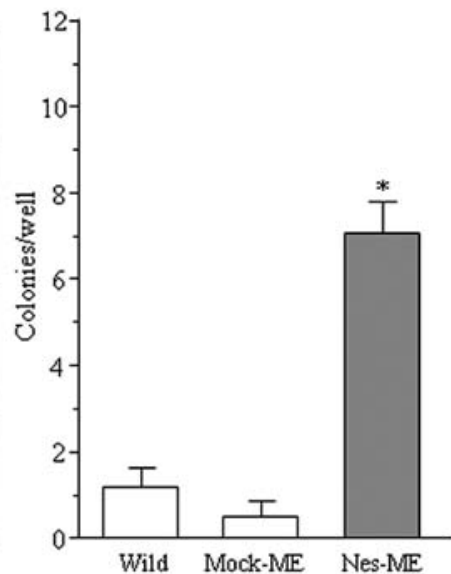

$\mathrm{C}$

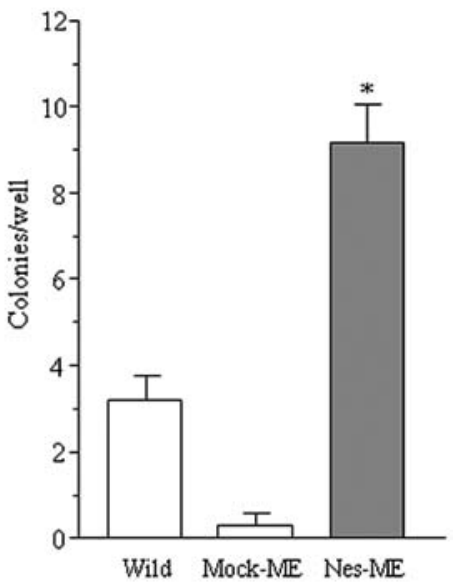

D
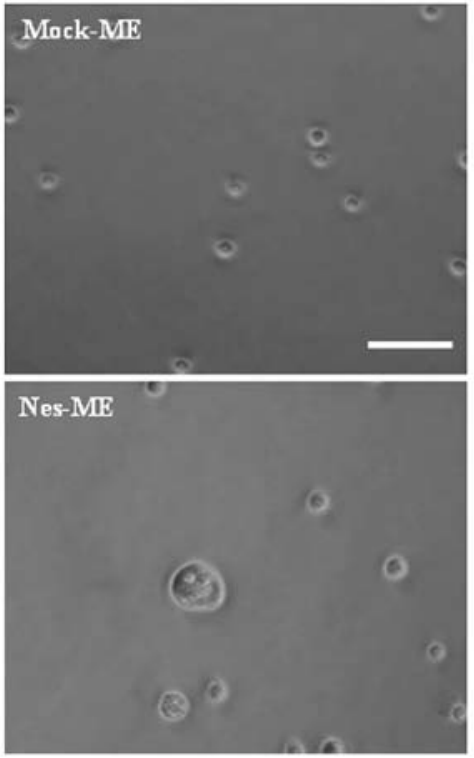

$\mathrm{E}$

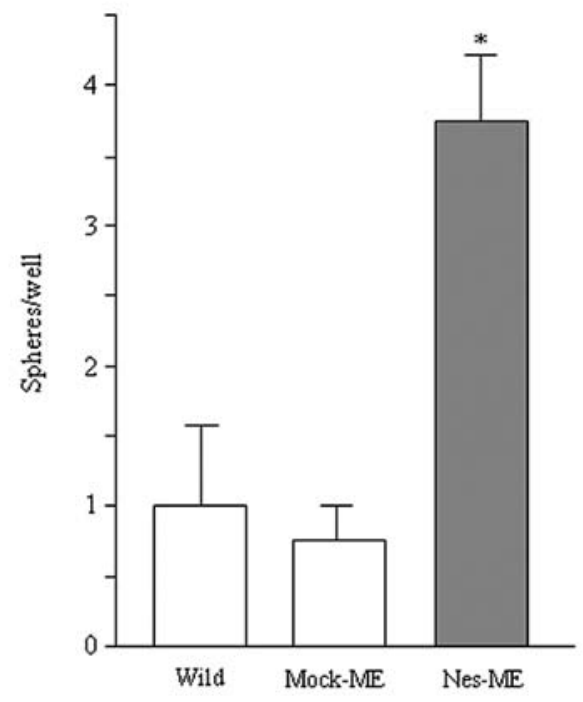

Figure 7. Anchorage-independent cell proliferation and sphere formation assays of nestin-transfected ME-180 cells. (A-C) In the soft agar assay, Nes-ME cells formed more colonies than Mock-ME and wild cells. (D) Nestin-transfected ME-180 cells formed larger and (E) more numerous spheres than mock cells. (D) Phase-contrast images: bar, $100 \mu \mathrm{m} ;{ }^{*} \mathrm{P}<0.0001$. 


\section{Discussion}

In this study, nestin was absent or only faintly expressed in normal squamous epithelial cells. Nestin expression was originally identified in neuroepithelial stem cells and neural cells (5). In pathological conditions, nestin is expressed in repair processes in the CNS, muscle, liver (44-47) and infarcted myocardium (48). In adult organisms, nestin-expressing cells are restricted to defined locations, where they may function as a cellular 'reserve' capable of proliferation, differentiation and migration after reactivation $(49,50)$. We found that nestin was slightly to moderately expressed in cervical hyperplasia and squamous metaplasia lesions of chronic cervicitis (data not shown). These findings suggest that nestin does not affect squamous cell functions in normal cervical tissues, but there is a possibility that nestin plays a role in reserve cells active in cervical inflammation.

Nestin expression levels correlated with CIN stage. A significant positive correlation exists between VEGF-A, VEGF-C, VEGF-D and VEGFR-3 expression and stage of cervical carcinogenesis (51). CIN 2,3 exhibited higher VEGF mRNA, transforming growth factor (TGF)- $\beta 1$ and TGF- $\beta$ receptor 1 levels than CIN 1 (52). Expression of insulin-like growth factor 1 receptor (IGF-1R) and phosphorylated IGFR-1 positively correlated with CIN grade (53). Our group reported that fibroblast growth factor receptor 2 IIIc (FGFR2 IIIc) expression was increased in advanced CIN stage (4). In the current study, the nestin expression pattern correlated with CIN stage, similar to the above-mentioned growth factors and receptors. Concerning precancerous lesions, activation of oncogenic K-ras in the nestin cell lineage in pancreatic tissue is sufficient for initiation of premalignant pancreatic intraepithelial neoplasia (PanIN) lesions in mice. These findings may indicate that nestin expression is closely related to the formation of precancerous lesions including the growth of neoplastic cells in CIN and carcinogenesis. Further research is needed to clarify the roles of nestin in precancerous lesions and carcinogenesis.

Previously, we found nestin immunoreactivity within cancer cells in $\sim 30 \%$ of pancreatic ductal adenocarcinoma cases (6). In prostate cancer, nestin is expressed in $75 \%$ of lethal androgen-independent prostate cancer cases (27). Among breast cancer subtypes, nestin is highly expressed in the basal subtype (ER $\left.\alpha^{-} / \mathrm{PR}^{-} / \mathrm{Her} 2^{-}\right)$, but not in the Her2 subtype (ER $\alpha^{-} /$ $\left.\mathrm{PR}^{-} / \mathrm{Her} 2^{+}\right)$or luminal epithelial subtype $\left(\mathrm{ER}^{+} / \mathrm{PR}^{+}\right)(26)$. In contrast to these malignant tumors, nestin was expressed in all cervical cancer cases, unrelated to their histological type. Although the etiology of nestin expression pattern in cervical cancer is unclear, we suggest that nestin may play a fundamental and important role in cervical carcinogenesis.

The CSC hypothesis suggests that only CSCs within the tumor can self-renew and proliferate extensively to form new tumors (54) and that CSCs are considered to be an attractive target for advanced cancer therapy (55). In cervical cancer, as in other cancers, characterization of CSCs will allow for the development of new treatments that are specifically targeted against this critical population of cells, particularly their ability to self-renew, resulting in more effective therapies (54). Previous studies suggested that $\mathrm{CD} 44^{+} \mathrm{CK} 17^{+} /$sphere-forming cervical cancer cells display stem cell properties (54). Using a sphere culture method that favors the growth of self-renewing cells, sphere-forming cells of cervical cancer showed an expression pattern of CD $44^{\text {high }} / \mathrm{CD} 24^{\text {low }}$ that resembles the CSC surface biomarker of breast cancer (55). Non-adherent spheres of cervical cancer maintained or increased ALDH and stem cell markers such as Nanog, nestin and Oct4 (38). Cells with self-renewal capability are able to sustain growth in suspension giving rise to non-adherent colonies (38). These results suggest that expression levels of nestin in cervical cancer cells closely correlate with CSC function.

In summary, nestin expression correlates with CIN progression and was expressed in all cervical cancer specimens examined. Furthermore, nestin stimulated colony and sphere formation in vitro. These findings suggest that nestin plays important roles in carcinogenesis and tumor formation of cervical cancer cells through regulation of CSC functions.

\section{Acknowledgements}

We express our appreciation to Ms. Yoko Kawamoto, Taeko Suzuki and Kiyoko Kawahara (Department of Pathology, Nippon Medical School, Tokyo, Japan) for their excellent technical assistance. We also thank Dr Shin-Ichi Tsuchiya (Division of Surgical Pathology, Nippon Medical School Hospital, Tokyo, Japan) for preparing tissue blocks.

\section{References}

1. Phongsavan K, Phengsavanh A, Wahlstrom R and Marions L: Women's perception of cervical cancer and its prevention in rural Laos. Int J Gynecol Cancer 20: 821-826, 2010.

2. Wang X, Liu R, Ma B, et al: High dose rate versus low dose rate intracavity brachytherapy for locally advanced uterine cervix cancer. Cochrane Database Syst Rev: CD007563, 2010.

3. Agarwal SM, Raghav D, Singh H and Raghava GP: CCDB: a curated database of genes involved in cervix cancer. Nucleic Acids Res 39: D975-D979, 2011.

4. Kawase R, Ishiwata T, Matsuda Y, et al: Expression of fibroblast growth factor receptor 2 IIIc in human uterine cervical intraepithelial neoplasia and cervical cancer. Int J Oncol 36: 331-340, 2010.

5. Lendahl U, Zimmerman LB and McKay RD: CNS stem cells express a new class of intermediate filament protein. Cell 60: 585-595, 1990.

6. Kawamoto M, Ishiwata T, Cho K, et al: Nestin expression correlates with nerve and retroperitoneal tissue invasion in pancreatic cancer. Hum Pathol 40: 189-198, 2009.

7. Ishiwata T, Teduka K, Yamamoto T, Kawahara K, Matsuda Y and Naito Z: Neuroepithelial stem cell marker nestin regulates the migration, invasion and growth of human gliomas. Oncol Rep 26: 91-99, 2011.

8. Marvin MJ, Dahlstrand J, Lendahl U and McKay RD: A rod end deletion in the intermediate filament protein nestin alters its subcellular localization in neuroepithelial cells of transgenic mice. J Cell Sci 111: 1951-1961, 1998.

9. Sjoberg G, Edstrom L, Lendahl U and Sejersen T: Myofibers from Duchenne/Becker muscular dystrophy and myositis express the intermediate filament nestin. J Neuropathol Exp Neurol 53: 416-423, 1994.

10. Steinert PM, Chou YH, Prahlad V, et al: A high molecular weight intermediate filament-associated protein in BHK-21 cells is nestin, a type VI intermediate filament protein. Limited co-assembly in vitro to form heteropolymers with type III vimentin and type IV alpha-internexin. J Biol Chem 274: 98819890, 1999.

11. Sejersen T and Lendahl U: Transient expression of the intermediate filament nestin during skeletal muscle development. J Cell Sci 106: 1291-1300, 1993.

12. Frojdman K, Pelliniemi LJ, Lendahl U, Virtanen I and Eriksson JE: The intermediate filament protein nestin occurs transiently in differentiating testis of rat and mouse. Differentiation 61: 243-249, 1997. 
13. Terling C, Rass A, Mitsiadis TA, Fried K, Lendahl U and Wroblewski J: Expression of the intermediate filament nestin during rodent tooth development. Int J Dev Biol 39: 947-956, 1995.

14. Kachinsky AM, Dominov JA and Miller JB: Myogenesis and the intermediate filament protein, nestin. Dev Biol 165: 216-228, 1994.

15. Ishizaki M, Ishiwata T, Adachi A, et al: Expression of nestin in rat and human glomerular podocytes. J Submicrosc Cytol Pathol 38: 193-200, 2006.

16. Yang J, Bian W, Gao X, Chen L and Jing N: Nestin expression during mouse eye and lens development. Mech Dev 94 287-291, 2000.

17. Almazan G, Vela JM, Molina-Holgado E and Guaza C: Re-evaluation of nestin as a marker of oligodendrocyte lineage cells. Microsc Res Tech 52: 753-765, 2001.

18. Amoh Y, Li L, Yang M, et al: Nascent blood vessels in the skin arise from nestin-expressing hair-follicle cells. Proc Natl Acad Sci USA 101: 13291-13295, 2004.

19. Lardon J, Rooman I and Bouwens L: Nestin expression in pancreatic stellate cells and angiogenic endothelial cells. Histochem Cell Biol 117: 535-540, 2002.

20. Takano T, Rutka JT and Becker LE: Overexpression of nestin and vimentin in ependymal cells in hydrocephalus. Acta Neuropathol 92: 90-97, 1996

21. Eaker EY and Sallustio JE: The distribution of novel intermediate filament proteins defines subpopulations of myenteric neurons in rat intestine. Gastroenterology 107: 666-674, 1994.

22. Yamada H, Takano T, Ito Y, et al: Expression of nestin mRNA is a differentiation marker in thyroid tumors. Cancer Lett 280 61-64, 2009

23. Strojnik T, Rosland GV, Sakariassen PO, Kavalar R and Lah T: Neural stem cell markers, nestin and musashi proteins, in the progression of human glioma: correlation of nestin with prognosis of patient survival. Surg Neurol 68: 133-144, 2007.

24. Brychtova S, Fiuraskova M, Hlobilkova A, Brychta T and Hirnak J Nestin expression in cutaneous melanomas and melanocytic nevi. J Cutan Pathol 34: 370-375, 2007.

25. Tsujimura T, Makiishi-Shimobayashi C, Lundkvist J, et al: Expression of the intermediate filament nestin in gastrointestinal stromal tumors and interstitial cells of Cajal. Am J Pathol 158: $817-823,2001$

26. Li H, Cherukuri P, Li N, et al: Nestin is expressed in the basal/ myoepithelial layer of the mammary gland and is a selective marker of basal epithelial breast tumors. Cancer Res 67 501-510, 2007.

27. Kleeberger W, Bova GS, Nielsen ME, et al: Roles for the stem cell associated intermediate filament Nestin in prostate cancer migration and metastasis. Cancer Res 67: 9199-9206, 2007.

28. Dahlstrand J, Collins VP and Lendahl U: Expression of the class VI intermediate filament nestin in human central nervous system tumors. Cancer Res 52: 5334-5341, 1992.

29. Ma YH, Mentlein R, Knerlich F, Kruse ML, Mehdorn HM and Held-Feindt J: Expression of stem cell markers in human astrocytomas of different WHO grades. J Neurooncol 86: 31-45, 2008

30. Klein WM, Wu BP, Zhao S, Wu H, Klein-Szanto AJ and Tahan SR: Increased expression of stem cell markers in malignant melanoma Mod Pathol 20: 102-107, 2007.

31. Florenes VA, Holm R, Myklebost O, Lendahl U and Fodstad O: Expression of the neuroectodermal intermediate filament nestin in human melanomas. Cancer Res 54: 354-356, 1994.

32. Liu C, Chen B, Zhu J, et al: Clinical implications for nestin protein expression in breast cancer. Cancer Sci 101: 815-819, 2010.

33. Matsuda Y, Naito Z, Kawahara K, Nakazawa N, Korc M and Ishiwata T: Nestin is a novel target for suppressing pancreatic cancer cell migration, invasion and metastasis. Cancer Biol Ther 11: 512-523, 2011.

34. Guzman-Ramirez N, Voller M, Wetterwald A, et al: In vitro propagation and characterization of neoplastic stem/progenitorlike cells from human prostate cancer tissue. Prostate 69: 1683-1693, 2009
35. Kasper S: Exploring the origins of the normal prostate and prostate cancer stem cell. Stem Cell Rev 4: 193-201, 2008

36. Hombach-Klonisch S, Paranjothy T, Wiechec E, et al: Cancer stem cells as targets for cancer therapy: selected cancers as examples. Arch Immunol Ther Exp (Warsz) 56: 165-180, 2008.

37. Okuno K, Ohta S, Kato H, Taga T, Sugita K and Takeuchi Y: Expression of neural stem cell markers in malignant rhabdoid tumor cell lines. Oncol Rep 23: 485-492, 2010.

38. Bortolomai I, Canevari S, Facetti I, et al: Tumor initiating cells: development and critical characterization of a model derived from the A431 carcinoma cell line forming spheres in suspension. Cell Cycle 9: 1194-1206, 2010.

39. Singh SK, Clarke ID, Hide T and Dirks PB: Cancer stem cells in nervous system tumors. Oncogene 23: 7267-7273, 2004.

40. Bentivegna A, Conconi D, Panzeri E, et al: Biological heterogeneity of putative bladder cancer stem-like cell populations from human bladder transitional cell carcinoma samples. Cancer Sci 101: 416-424, 2010.

41. Jimeno A, Feldmann G, Suarez-Gauthier A, et al: A direct pancreatic cancer xenograft model as a platform for cancer stem cell therapeutic development. Mol Cancer Ther 8: 310-314, 2009.

42. Kurban G, Ishiwata T, Kudo M, Yokoyama M, Sugisaki Y and Naito Z: Expression of keratinocyte growth factor receptor (KGFR/FGFR2 IIIb) in human uterine cervical cancer. Oncol Rep 11: 987-991, 2004.

43. Naito Z, Ishiwata T, Kurban G, et al: Expression and accumulation of lumican protein in uterine cervical cancer cells at the periphery of cancer nests. Int J Oncol 20: 943-948, 2002.

44. Aarimaa V, Kaariainen M, Vaittinen S, et al: Restoration of myofiber continuity after transection injury in the rat soleus. Neuromuscul Disord 14: 421-428, 2004.

45. Holmin S, Almqvist P, Lendahl U and Mathiesen T: Adult nestin-expressing subependymal cells differentiate to astrocytes in response to brain injury. Eur J Neurosci 9: 65-75, 1997.

46. Lin RC, Matesic DF, Marvin M, McKay RD and Brustle O: $\mathrm{Re}$-expression of the intermediate filament nestin in reactive astrocytes. Neurobiol Dis 2: 79-85, 1995.

47. Niki T, Pekny M, Hellemans K, et al: Class VI intermediate filament protein nestin is induced during activation of rat hepatic stellate cells. Hepatology 29: 520-527, 1999.

48. El-Helou V, Dupuis J, Proulx C, et al: Resident nestin+ neurallike cells and fibers are detected in normal and damaged rat myocardium. Hypertension 46: 1219-1225, 2005.

49. Wiese C, Rolletschek A, Kania G, et al: Nestin expression - a property of multi-lineage progenitor cells? Cell Mol Life Sci 61: 2510-2522, 2004

50. Teranishi N, Naito Z, Ishiwata T, et al: Identification of neovasculature using nestin in colorectal cancer. Int J Oncol 30 593-603, 2007

51. Hammes LS, Tekmal RR, Naud P, et al: Up-regulation of VEGF, c-fms and COX-2 expression correlates with severity of cervical cancer precursor (CIN) lesions and invasive disease. Gynecol Oncol 110: 445-451, 2008.

52. Soufla G, Sifakis S, Baritaki S, Zafiropoulos A, Koumantakis E and Spandidos DA: VEGF, FGF2, TGFB1 and TGFBR1 mRNA expression levels correlate with the malignant transformation of the uterine cervix. Cancer Lett 221: 105-118, 2005.

53. Kuramoto H, Hongo A, Liu YX, et al: Immunohistochemical evaluation of insulin-like growth factor I receptor status in cervical cancer specimens. Acta Med Okayama 62: 251-259, 2008.

54. Feng D, Peng C, Li C, et al: Identification and characterization of cancer stem-like cells from primary carcinoma of the cervix uteri. Oncol Rep 22: 1129-1134, 2009.

55. Gu W, Yeo E, McMillan N and Yu C: Silencing oncogene expression in cervical cancer stem-like cells inhibits their cell growth and self-renewal ability. Cancer Gene Ther 18: 897-905, 2011. 